\title{
Autogenous Tooth Bone Graft - A Biomimetic Promise for Regenerative Dentistry
}

\section{Neha Dilip Bhalla ${ }^{1 *}$, Sudhir R Patil ${ }^{2}$, Sphoorthi A Belludi ${ }^{3}$, Ashwin Prabhu', Veena HR$^{4}$, Sneha Dani ${ }^{5}$ and Apeksha Birla $^{6}$}

${ }^{1}$ Consultant Periodontist and Oral Implantologist, M.L. Dhawale Rural Homeopathic Hospital, Palghar, Maharashtra, India

${ }^{2}$ Ex-Professor and Head, Department of Periodontics, K.L.E Society's Institute of Dental Sciences and Hospital, Karnataka, India

${ }^{3}$ Professor and Head-Department of Periodontics, K.L.E Society's Institute of Dental Sciences and Hospital, Karnataka, India

${ }^{4}$ Reader, Department of Periodontics, K.L.E Society's Institute of Dental Sciences and Hospital, Karnataka, India

${ }^{5}$ Senior lecturer, Department of Periodontics, K.L.E Society's Institute of Dental Sciences and Hospital, Karnataka, India

${ }^{6}$ IIIrd Year Post Graduate Student - Department of Periodontics and Oral Implantology, SMBT Dental College and Postgraduate Research Centre, Sangamner, Maharashtra, India

*Corresponding Author: Neha Dilip Bhalla, Consultant Periodontist and Oral Implantologist, M.L. Dhawale Rural Homeopathic Hospital, Palghar, Maharashtra, India.

Received: July 02, 2019; Published: August 23, 2019

DOI: $10.31080 /$ ASDS.2019.03.0622

\begin{abstract}
Regeneration is defined as reconstitution of a lost or injured part, with form and function of lost structures restored. Graft associated regeneration still remains the mainstay and newer graft materials of diverse origin are added to the already existing lot, with claims of superior clinical outcomes. Thus, the search for newer, near ideal biomaterials and procedures continue to be a challenge in the field of regenerative dentistry. Based on the regenerative properties of osteoconduction, osteoinduction, creeping substitution and osteogenesis mediated via the expression of growth factors derived from the patient's own tooth and the histo-morphological similarities between tooth and bone, a novel bone graft material has been developed utilizing the structural components of an extracted tooth. This review elaborately describes a novel regenerative technology of the autogenous tooth derived bone graft material, a biomimetic system that treats patients by fabricating a bone graft material from their own extracted teeth.
\end{abstract}

Keywords: Autogenous Tooth Bone Graft; Bone Regeneration, Bone Substitutes; Endogenous Regenerative Technology; Tooth

\section{Introduction}

Regeneration is defined as reconstitution of a lost or injured part, with form and function of lost structures restored [1]. Periodontal Regeneration comprises de novo osteogenesis, cementogenesis and generation of functionally oriented periodontal fibers into both newly formed alveolar bone and cementum. Periodontal Regeneration constitute a complex multifactorial process influenced by interaction among various progenitor cells, hormones, growth factors, and the components of the extra cellular matrix [2]. Several different approaches are currently under research and exploration to achieve complete, reliable, and reproducible results in regeneration of periodontal tissues. The therapeutic management of the interosseous periodontal defects is the formation of new bone for successful periodontal regeneration.

Over the past five decades, attempts at periodontal regeneration are harnessed with an array of biomaterials which include Bone graft materials of various types, Guided tissue regeneration (GTR) membranes, biologics like Enamel matrix derivatives (EMD), Growth factors (GF), Bone morphogenic proteins (BMP) and Platelet concentrate analogues. The emergence of tissue 
engineering concepts has further opened a new exciting arena in periodontal regeneration.

Graft associated regeneration still remains a mainstay and newer graft materials of diverse origin are added to the already existing lot, with claims of superior clinical outcomes. Autogenous bone grafts have been regarded as gold standard for the reconstruction of hard tissue defects with promising osteogenic, osteoconductive, osteoinductive, and osseointegrative capabilities without triggering any foreign body reaction in the host. However, due to difficulties faced in procuring them in an adequate amount along with the plausible risk of donor site morbidity pose as challenges [3]. This result in a surge for research on the development, application, and effectiveness of non-autologous origin grafting materials namely allografts, xenografts and synthetic grafts. But these too are associated with problems of antigenicity, immune rejection response by heterologous proteins, contagion possibility and high cost. It is therefore, difficult to obtain bone graft material with the advantages of autogenous bone but not its disadvantages [4]. Thus, the search for newer, near ideal biomaterials and procedures continue to be a challenge in the field of regenerative periodontics.

\section{Tooth and bone}

Teeth are known as a composite of organic and inorganic components consisting of minerals of the calcium phosphate range, collagen and other organic elements. The compositions of teeth and bone are very chemically similar. The tooth which is composed of inorganic and organic ingredients within the dentin has a $65 \%$ : $35 \%$ ratio, cementum of $45-50 \%: 50-55 \%$ and the alveolar bone of $65 \%: 35 \%$, respectively [5]. Thereby, sharing similar bone remodeling potential and histo-morphological resemblance to the host bone. Based on the regenerative forte of a tooth by the processes of osteoconduction, osteoinduction, creeping substitution and osteogenesis via recruitment of growth factors, a novel bone graft material has been developed utilizing the structural components of an extracted tooth [6].

Herewith, this review elaborately describes the autologous bone grafting technology of the autogenous tooth derived bone graft material, a biomimetic regenerative system that treats patients by manufacturing a bone graft material from their own extracted teeth.
Development of autogenous tooth bone graft material

The use of bone grafts in the management of periodontal intraosseous defects was proposed in studies by Hegedus in 1923. A major revolution has resulted from then to the current practice in the evolution of these bone grafts. Ike and Urist suggested recycled root dentin prepared from extracted teeth for use as a carrier of Recombinant human BMP-2 (rh-BMP2) because it induces new bone formation in the periodontium [7].

It was, however, the breakthrough research done by Kim YK et al. which proved the successful use of artificially processed tooth as a bone grafting material [5]. Murata., et al. further supported that demineralized dentin matrix (DDM) does not inhibit BMP2 activity rather shows a better release profile of BMP-2 [8]. Chung PH extracted proteins from teeth in their papers published in 2002 and 2004, provided evidence that teeth contain BMP, further strengthening the role $[9,10]$.

Patient's extracted tooth is classified as environmental waste. However, the use of tooth may be permitted if the patient consents to process and use his/her own teeth. Reports even suggest that the remainder of a root can be left intentionally to preserve the alveolar bone [11,12]. Literature also reported that $90 \%$ of the tooth's organic components are type I collagen and has growth factors such as Insulin-like growth factor (IGF)-II, BMP2, and transforming growth factor (TGF)-beta like bone, all of these playing a crucial role in the calcification of bone [13]. Studies also suggest that the tooth enamel expressed BMP2 which possess osteoinductive potential $[14,15]$. Even the tooth cement and its progenitor cell contain TGF-beta, IGF-I and platelet derived growth factor, which are also essential for bone formation [16]. To summarize, the tooth borne enamel and cement have many growth factors and proteins that are vital for bone formation.

Biocompatibility of bone graft material using teeth

Studies have been conducted since 1993, Kim YK., et al. have conducted basic studies such as component analysis, research through electron microscope and identified the main component of the toothash powder to be Hydroxyapatite (HA) and betaTricalcium phosphate ( $\beta$-TCP), which was osteoconductive as well as biocompatible and absorbed over time $[17,18]$. Since then, the results of many related experimental studies have suggested 
the indications and comparisons for guided bone regeneration using lyodura and with other bone substitutes, tissue reaction after implantation, cytotoxicity, hypersensitivity test and biocompatibility have been evaluated [19-23].

\section{Osteoinductivity and osteo conductivity of teeth}

A variety of research has been performed for the comparison with other bone grafts like xenograft, the evaluation of their healing process around implants, along with platelet analogues like platelet rich plasma, the healing process after inducing osteoporosis, multiple application of tissue adhesive, chitosan, and the healing process after guided bone regeneration. Tooth ash has proven to be an osteoconductive bone graft material with excellent biocompatibility in evidenced literature [24-28].

Bessho K., et al. extracted human dentin matrix and found in Sodium dodecyl sulphate polyacralamide gel electrophoresis (SDS-PAGE) and Isoelectric focusing (IEF) that purified BMP is homogenous, inducing the formation of new bone within 3 weeks of implantation in muscle pouches in Wistar rats [29]. Also influence of dentin matrix derived BMP is very similar to bone matrix derived BMP, both exhibiting the same action in the body [30].

Boden SD., et al. suggested that LIM mineralization protein 1 (LMP-1) is pivotal for regulation of osteoblastic differentiation and their maturation leading to bone formation [31]. Their presence was confirmed by Wang X., et al. wherein LIM expression was evident in the predentin layer of the odontoblastic and endothelial cells of the teeth [32].

Further in an ex vivo study performed on artificially created alveolar bone defects in Wistar rats were filled with either tooth or iliac bone graft material or left as controls. The study concluded that tooth graft material produced a similar amount of new bone compared to iliac bone graft material at the end of 8 weeks. Dentin sialoprotein was found to be expressed in the new bone formed by the tooth graft material at 6 weeks. The authors also suggested as early as 6-8 weeks for new bone formation as compared to the conventional bone graft [33].

Similar results were found in a histomorphometric study by Jeong HR on miniature pigs for bone regeneration in an artificial bony defect. They examined the effect of autogenous tooth bone as a graft material which showed higher amount of bone formation and remodeling in experimental groups and concluded the usefulness of this graft with possible clinical feasibility in the reconstruction of bony defects [34].

A prospective study was conducted to evaluate the bone healing effect and clinical capabilities like bone healing ability of autogenous tooth bone materials as a scaffold when used together with growth factor in patients with alveolar deficient sites for implant placement [35].

An analytical study of extracted fresh tooth, X-ray diffraction (XRD) pattern of dentin of autogenous tooth bone graft material was found to be most similar to autogenous bone [36].

In a study performed quantitative analysis of the proliferation and differentiation of the MG63 cell line on the bone grafting material using human tooth demonstrated that the cellular adhesion and proliferation activity of MG63 cells on partially demineralized dentin matrix (PDDM) were comparable and could be controlled with enhanced osteogenic differentiation [37].

\section{Clinical application of autogenous tooth bone graft material}

Endogenous regenerative technology and autogenous tooth bone graft in periodontal regeneration

Based on the principles of endogenous regenerative technology (ERT), stimulation of latent self-repair mechanisms in patients and harnessing the host's innate capacity using the patient's own "Regenerative Tools", i.e. Patient-derived growth factors and fibrin scaffolds to create a material niche in an injured site where the progenitor/stem cells from neighboring tissues can be recruited for in situ periodontal regeneration [38]. Fulfilling this criteria, the patient's tooth derived autogenous bone grafts, like the Demineralized dentin matrix (DDM) and Demineralized bone matrix (DBM) composed of predominantly type I collagen (95\%) and matrix binding proteins such as BMPs namely transforming growth factor-beta (TGF- $\beta$ ), insulin growth factor-I (IGF-I) and IGFII were detected in human dentin [39-42]. Thus, contributing in the role of promoting bone resorption and bone formation [43-45]. Therefore, these tooth derived bone graft materials are considered to be potentially useful in periodontal regenerative sciences in management of interosseous defects.

\section{Alveolar cleft repair}

A report published on the application of a combination of maxillary bone transport and auto tooth bone grafting for alveolar 
cleft repair using autogenous extracted teeth, concluded to be an effective approach for alveolar cleft repair. It was seen that Autotooth bone allowed for repair of the bone defects of the nasal floor and alveolar cleft and resulted in a superior bone connection [46].

Maxillary sinus and ridge augmentation

In an another in-vivo study performed on 250 patients, Implant placement was combined with maxillary sinus augmentation using block type, powder type, and block with powder type autobone graft material. In their histological assessment, formation of new bone, densified lamellated bone, trabecular bones, osteoblast and planting fixtures were investigated [4].

Similar results were confirmed by Jeong KI study wherein it was found appropriate for the use in maxillary sinus augmentation [47].

A prospective study was performed to evaluate the effectiveness of two bone graft materials in the application for sinus bone graft procedure, using Bio-Oss in control and autogenous tooth bone graft (AutoBT) powder in experimental group respectively. There was significant difference in osteoid thickness $(8.35 \mu \mathrm{m}$ in Bio-0ss group Vs. $13.12 \mu \mathrm{m}$ in AutoBT group) $(\mathrm{P}=0.025)$. Thus concluding that AutoBT could be considered a viable alternative to the autogenous bone or other bone graft materials in sinus bone graft procedure [48].

Current consensus suggests autogenous teeth bone grafting for alveolar ridge augmentation to be a clinically safe with good bone forming capacity, and shows good outcome on implant stability [49].

\section{Guided bone regeneration (GBR)}

A number of clinical studies comparatively analyzed the clinical results for vertical and horizontal ridge augmentation (GBR) using the autogenous tooth bone graft material, with and without the resorbable membrane. The results showed clinically acceptable bone regeneration without any eventful complications [50] with excellent clinical results were evaluated [51].

In a clinical experimental study which conducted a Feasibility Analysis in humans concluded that the autogenous tooth-based bone graft (ATBBG) material was not only viable for GBR but also yielded a stable marginal bone level (MBL) even after functional loading of implants [52].
A clinical study evaluated the efficacy of the autogenous tooth bone graft material, clinically and radiologically, as related to implant installation with various surgical procedures like guided bone regeneration (GBR), sinus graft and defect filling using autogenous tooth bone. The histological assessment revealed, formation of the new bone and continuous trabecular bone pattern identified around autogenous tooth bone. Thus suggesting that this material as a potential substitute for the autogenous bone [53].

\section{Socket preservation}

In a human study conducted by Gomes., et al. the experimental sockets were filled with autogenous DDM and covered with polytetrafluoroethylene (PTFE) membrane. These sockets showed bone formation of the same radiopacity as the surrounding bone, at a faster rate and superior bony architecture as compared to the other groups [54]. Similarly supporting studies published by Kim., et al. and Park SM., et al. also reported good healing of the extraction socket after 3 to 3.5 months for future implant placement after autogenous tooth bone graft placement [55].

\section{Endodontic applications}

Work by Jiang Y., et al reported the use of autogenous DDM as a prospective material of choice as an apexification material as well as a permanent root canal filling material in the branch of endodontics [56].

Other applications

The tooth derived Autogenous bone graft may serve as a potential bone grafting material for oral pathological conditions like resected tumors, enucleated cyst, traumatic injuries resulting in pathological fracture predisposition. Autogenous DDM has further been reported to be an ideal scaffold for stem cells and bone growth factors and found emerging acceptance in endodontic and restorative dentistry [5].

\section{Conclusion}

Extracted tooth are categorized as a hazardous biomedical waste and disposed appropriately. However, if the same extracted teeth can be processed into a potential regenerative biomaterial and used in the same patient for his/her oral health benefit, it may serve as an exciting and novel approach in regenerative sciences. The emergence of autogenous tooth bone graft based on the principles of Endogenous Regenerative Technology (ERT) promotes not only the natural healing process but also reconstruction of 
lost tissues by body's inherent capabilities. Thus, Randomized clinical trials comparing this biomimetic material with other commercially available bone graft materials are the need of the hour to substantiate its purposefulness.

\section{Conflict of Interest}

The authors have none to declare.

\section{Bibliography}

1. "Glossary of periodontal terms". In: 3rd ed. Chicago (IL): American Academy of Periodontology American Academy of Periodontology (1992).

2. Subramaniam MR., et al. "Bone Morphogenetic Proteins: Periodontal Regeneration". North American journal of medicine and science 5.3 (2013): 161-168.

3. Zeichner-David M. "Regeneration of periodontal tissues: Cementogenesis revisited". Periodontology 41 (2006): 196-217.

4. Park SM., et al. "Clinical application of auto-tooth bone graft material". Journal of the Korean Association of Oral and Maxillofacial Surgeons 38.1 (2012): 2-8.

5. Kim YK., et al. "Development of a novel bone grafting material using autogenous teeth". Oral Surgery, Oral Medicine, Oral Pathology and Oral Radiology 109.4 (2010): 496-503.

6. Kim YK., et al. "Tooth-derived bone graft material". Journal of the Korean Association of Oral and Maxillofacial Surgeons 39.3 (2013): 103-111.

7. Kumar J., et al. "Journey of bone graft materials in periodontal therapy: A chronological review". Journal of Dental and Allied Sciences 5.1 (2016): 30-34.

8. Murata M., et al. "Human dentin as novel biomaterial for bone regeneration". Biomaterials Physics and Chemistry (2011): 127-140.

9. Chung PH Korea Intellectual Property Rights Information Service, assignee. Method for extracting tooth protein from extracted tooth. Korea patent. KR 1020020008789. (2004).

10. Chung PH Korea Intellectual Property Rights Information Service, assignee. Tooth protein extracted from extracted tooth and method for using the same. Korea patent. KR 1020040051812. (2005).
11. Gongloff RK. "Vital root retention". International Journal of Oral and Maxillofacial Surgery 3 (1974): 97-99.

12. Fareed K., et al. "Vital root retention: a clinical procedure". Journal of Prosthetic Dentistry 62.4 (1989): 430-434.

13. Kim SG., et al. "Combined implantation of particulate dentine, plaster of Paris, and a bone xenograft (Bio-Oss) for bone regeneration in rats". Journal of Cranio-Maxillofacial Surgery 29.5 (2001): 282-288.

14. Ike M and Urist MR. "Recycled dentin root matrix for a carrier of recombinant human bone morphogenetic protein". Journal of Oral Implantology 24.3 (1998): 124-132.

15. Goncalves EAL. "Study of the process of bone repair in surgical defects implanted with demineralized autogenous dentin matrix in radius of dog [thesis]". Bauru: University of Sao Paulo (1997).

16. Saygin NE., et al. "Growth factors regulate expression of mineral associated genes in cementoblasts". Journal of Periodontology 71.10 (2000): 1591-1600.

17. Kim YK., et al. "An experimental study on the tissue reaction of toothash implanted in mandible body of the mature dog". Journal of Korean Association of Maxillofacial Plastic and Reconstructive Surgeons 15.2 (1993): 129-136.

18. Kim YK., et al. "Implantation of toothash combined with plaster of Paris: experimental study". Journal of Korean Association of Maxillofacial Plastic and Reconstructive Surgeons 16.2 (1994): 130-136.

19. Kim YK. "The experimental study of the implantation of toothash and plaster of Paris and guided tissue regeneration using Lyodura". Journal of the Korean Association of Oral and Maxillofacial Surgeons 22.2 (1996): 297-306.

20. Kim YK and Yeo HH. "Transmitted electronic microscopic study about the tissue reaction after the implantation of toothash". Journal of the Korean Association of Oral and Maxillofacial Surgeons 23.2 (1997): 283-289.

21. Kim YK., et al. "An experimental study on the healing process after the implantation of various bone substitutes in the rats". Journal of the Korean Association of Oral and Maxillofacial Surgeons 27.1 (2001): 15-24. 
22. Kim YK., et al. "Cytotoxicity and hypersensitivity test of toothash". Journal of Korean Association of Maxillofacial Plastic and Reconstructive Surgeons 23.5 (2001): 391-395.

23. Kim SG., et al. "Grafting of large defects of the jaws with a particulate dentin plaster of Paris combination". Oral Surgery, Oral Medicine, Oral Pathology and Oral Radiology 88.1 (1999): 22-25.

24. Kim SG., et al. "Combined implantation of particulate dentine, plaster of Paris, and a bone xenograft (BioOss) for bone regeneration in rats". Journal of Cranio-Maxillofacial Surgery 29.5 (2001): 282-288.

25. Kim SG., et al. "Use of particulate dentin plaster of Paris combination with/without platelet rich plasma in the treatment of bone defects around implants". The International Journal of Oral and Maxillofacial Implants 17.1 (2002): 86-94.

26. Kim SY., et al. "Effects on bone formation in ovariectomized rats after implantation of tooth ash and plaster of Paris mixture". Journal of Oral and Maxillofacial Surgery 62.7 (2004): 852-857.

27. Park SS., et al. "Osteogenic activity of the mixture of chitosan and particulate dentin". Journal of Biomedical Materials Research Part A 87.3 (2008): 618-623.

28. Kim WB., et al. "Effect of Tisseel on bone healing with particulate dentin and plaster of Paris mixture”. Oral Surgery, Oral Medicine, Oral Pathology and Oral Radiology 109.2 (2010): e34-e40.

29. Bessho K., et al. "Purification of rabbit bone morphogenetic protein derived from bone, dentin, and wound tissue after tooth extraction". Journal of Oral and Maxillofacial Surgery 48.2 (1990): 162-169.

30. Bessho K., et al. "Human dentin matrix derived bone morphogenetic protein". Journal of Dental Research 70.3 (1991): 171175.

31. Boden SD., et al. "LMP1, a LIM domain protein, mediates BMP6 effects on bone formation". Endocrinology 139.12 (1998): 5125-5134.

32. Wang X., et al. "Immunohistochemical localization of LIM mineralization protein 1 in pulp dentin complex of human teeth with normal and pathologic conditions". Journal of Endodontics 34.2 (2008): 143-147.
33. Nampo T., et al. "A new method for alveolar bone repair using extracted teeth for the graft material". Journal of Periodontology 81.9 (2010): 1264-1272.

34. Jeong HR., et al. "Effectiveness of autogenous tooth bone used as a graft material for regeneration of bone in miniature pig". Journal of Korean Association of Maxillofacial Plastic and Reconstructive Surgeons 37.5 (2011): 375-379.

35. Ahn KJ., et al. "Effectiveness of Autogenous Tooth Bone Graft Combined with Growth Factor: Prospective Cohort Study". Journal of Korean Dental Science 6 (2013): 50-57.

36. Kim YK., et al. "Analysis of the inorganic component of autogenous tooth bone graft material". Journal of Nanoscience and Nanotechnology 11.8 (2011): 7442-7445.

37. Lee HJ. In: Quantitative analysis of proliferation and differentiation of MG63 cell line on the bone grafting material using human tooth [dissertation]. [Seoul]: Seoul National University 2011.

38. Fa-Ming Chen., et al. "A review on endogenous regenerative technology in periodontal regenerative medicine". Biomaterials 31.31 (2010): 7892-7927.

39. Murata M., et al. "Bone augmentation by onlay implant using recombinant human BMP-2 and collagen on adult rat skull without periosteum". Clinical Oral Implants Research 11.4 (2000): 289-295.

40. Akazawa T., et al. "Biodegradation and bioabsorption innovation of the functionally graded cattle bone-originated apatite with blood compatibility". Journal of Biomedical Materials Research 76.1 (2006): 44-51.

41. Murata M., et al. "Blood permeability of a novel ceramic scaffold for bone morphogenetic protein-2". Journal of Biomedical Materials Research 81.2 (2007): 469-475.

42. Finkelman., et al. "Quantitation of growth factors IGF-I, SGF/ IGF-II, and TGF-beta in human dentin". Journal of Bone and Mineral Research 5.7 (1990): 717-723.

43. Nanci A. "In: Ten cate's oral histology". 7th ed. Amsterdam: Elsevier Inc. 202-211.

44. Min BM. "In: Oral biochemistry". Seoul: Daehan Narae Pub Co (2007): 22-26.

45. Bhaskar SN. "In: Orban's oral histology and embryology". 9th ed. Saint Louis: Mosby Co (1980). 
46. Shingo H., et al. "Brief Clinical Studies Bone Transport and Bone Graft Using Auto-Tooth Bone for Alveolar Cleft Repair". Journal of Craniofacial Surgery 24.1 (2013): 65-68.

47. Jeong KI., et al. "Clinical study of graft materials using autogenous teeth in maxillary sinus augmentation". Implant Dentistry 20.6 (2011): 471-475.

48. Jun SH., et al. "A prospective study on the effectiveness of newly developed autogenous tooth bone graft material for sinus bone graft procedure". The Journal of Advanced Prosthodontics 6.6 (2014): 528-538.

49. Gual-Vaques P., et al. J. Med Oral Patol Oral Cir Buccal 23.1 (2018): 112-119

50. Lee JY., et al. "Comparative analysis of guided bone regeneration using autogenous tooth bone graft material with and without resorbable membrane". Journal of Dental Sciences 8.3 (2013): 281-286.

51. Lee JY., et al. "Clinical evaluation of ridge augmentation using autogenous tooth bone graft material: case series study". Journal of the Korean Association of Oral and Maxillofacial Surgeons 39.4 (2013): 156-160.

52. Hee-Yung C., et al. "Feasibility Analysis of Autogenous Toothbased Bone Graft Material after Guided Bone Regeneration Technique". Journal of Case Reports and Studies 2.3 (2014): 304.

53. Han MW and Lee JK. "Clinical Study on the Efficacy of the Autogenous Tooth Bone Graft Material (AutoBT)". Journal of Korean Association of Maxillofacial Plastic and Reconstructive Surgeons 35 (2013): 221-226.

54. Gomes MF., et al. "Densitometric analysis of the autogenous demineralized dentin matrix on the dental socket wound healing process in humans". Brazilian Oral Research 20.4 (2006): 324-330.
55. Kim YK., et al. "Extraction socket preservation and reconstruction using autogenous tooth bone graft:". Journal of Korean Association of Maxillofacial Plastic and Reconstructive Surgeons 33.3 (2011): 264-269.

56. Jiang Y., et al. "Clinical studies on apexification with demineralized dentin matrix". West China Journal of Stomatology 21.6 (2003): 460-462.

\section{Volume 3 Issue 9 September 2019}

(C) All rights are reserved by Neha Dilip Bhalla, et al. 